\title{
La medición del impacto de las innovaciones metodológicas sobre los resultados de la docencia universitaria
}

\author{
Alfredo Prieto Martín 1, *, David Díaz Martín ${ }^{2}$, Jorge Monserrat Sanz ${ }^{3}$ y José Barbarroja \\ Escudero ${ }^{4}$ \\ 1 Profesor contratado Doctor, Departamento de Medicina y Especialidades Médicas, Universidad de Alcalá; \\ https://orcid.org/0000-0002-4314-5889 \\ 2 Departamento de Medicina y Especialidades Médicas, Universidad de Alcalá; https://orcid.org/0000-0002- \\ 5672-6658 \\ 3 Departamento de Medicina y Especialidades Médicas, Universidad de Alcalá; https://orcid.org/0000-0003- \\ $1775-4645$ \\ 4 Departamento de Medicina y Especialidades Médicas, Universidad de Alcalá; https://orcid.org/0000-0002- \\ 8226-5357 \\ * Autor correspondencia: alfredo.prieto@uah.es
}

DOI: https://doi.org/10.37536/RIECS.2020.5.1.201

Recibido: 07/05/2020; Aceptado: 14/05/2020; Publicado: 30/05/2020

Resumen: El estudio del impacto de las innovaciones educativas sobre el aprendizaje producido en las asignaturas universitarias, requiere medir los efectos del cambio metodológico sobre diversas variables asociadas a la mejora del aprendizaje. En esta revisión mostramos e ilustramos cómo realizar una serie de medidas sobre algunas de estas variables: la implicación de los alumnos en la asignatura, el tiempo de estudio de los alumnos, el rendimiento académico (cuantificando las tasas de éxito y de fracaso), el aprendizaje (calificaciones medias obtenidas en las pruebas de evaluación del aprendizaje). El impacto sobre estas variables puede cuantificarse por el tamaño de efecto (g) que relaciona el nivel de cambio observado en la variable de estudio con respecto a su nivel de variación inicial estimado por su desviación estándar. Finalmente, desde la perspectiva del alumno es importante conocer cuál es su percepción y valoración sobre el cambio metodológico experimentado. En esta revisión aportamos ejemplos de casos que ilustran cómo pueden estudiarse los efectos del modelo de aula invertida sobre la implicación, el aprendizaje y la valoración de los alumnos sobre el cambio de metodología de enseñanza y el aprendizaje y mostramos los resultados que hemos obtenido. Utilizando estas metodologías demostramos que la puesta en práctica del aula inversa en asignaturas universitarias estimula el trabajo de los alumnos, aunque usualmente se requieren 2-3 años de práctica para vencer la resistencia estudiantil inicial y lograr su máximo impacto sobre el rendimiento académico, el aprendizaje y la apreciación de su valor por los alumnos. aula inversa, implicación estudiantil, rendimiento académico, tamaño de efecto, aprendizaje activo, implicación estudiantil, motivación, gamificación.

Palabras Clave: Aula inversa, Implicación estudiantil, Rendimiento académico, Tamaño de efecto, Aprendizaje activo, Implicación estudiantil, Motivación, Gamificación.

Abstract: The study of the impact of educational innovations on student learning in university
courses requires measurements of the effects of the methodological changes on several variables
associated to the enhancement of learning. In this review we show how to perform measurements
of the involvement of the students in the subject, the student study time, academic performance
(quantifying passing and failure rates), and the learning (changes in average grades on learning
assessment exams). The impact on these variables is quantified by the effect size (g) that relates the
level of change observed in the study variable with respect to its level of initial variation estimated
by its standard deviation. Finally, from a student's perspective it is important to know what the 
students' perception and assessment are about the methodological change experienced. In this work, we provide some examples of how can be studied the effect of the implementation of the inverted classroom model on the involvement, learning and assessment of students on the change in teaching methodology and learning, and we show the results we have obtained. Using these methodologies we demonstrate that the implementation of the flipped classroom in university courses stimulates the work of the students, although usually it takes 2-3 years of practice to overcome student resistance, to achieve its maximum impact on academic performance, student learning, and the student's appreciation of its value.

Key words: Adaptive Flipped Classroom, Student Engagement, Academic Performance, Effect Size, Active Learning, Motivation, Gamification.

\section{Introducción}

En todo proceso de innovación educativa es muy importante conocer el impacto de los cambios realizados, y para ello, es necesario planificar cómo se va a evaluar el efecto de la innovación sobre el trabajo de los alumnos, su aprendizaje, su rendimiento académico y su percepción de la experiencia de aprendizaje y la docencia recibida. En el modelo de aula invertida o flipped classroom el profesor crea un entorno online en el que sus alumnos tienen la responsabilidad de contactar con la información a aprender antes de que esta sea tratada en clase [1]. Para ello el docente fomenta el estudio preparatorio de sus alumnos mediante diversas formas de evaluación continua adelantada [2]. Al disponer de alumnos preparados y del feedback procedente de éstos, sobre sus dificultades para comprender las ideas esenciales, el docente puede enfocar las actividades de clase en la resolución de las dificultades manifestadas por sus alumnos [3].

Nuestra metodología de aula inversa adaptativa se basa fundamentalmente en el uso del just in time teaching (JITT) [4], aunque lo combinamos con el uso de otros métodos de fomento del estudio preparatorio para las clases mediante evaluación formativa como son el team based learning (TBL) [4] y el peer instruction (PI) [5]. Esta combinación de metodologías la hemos denominado aula invertida adaptativa porque la comunicación bidireccional entre el docente y sus alumnos antes de la clase proporciona al docente información veraz con la que podrá enfocarse en la resolución de las dificultades y necesidades comunicadas por sus estudiantes. Con esta combinación de innovaciones metodológicas pretendemos provocar un cambio (que es muy necesario y beneficioso) en los hábitos de estudio de nuestros alumnos y una mejor comprensión por parte del profesor de las dificultades de sus alumnos para comprender y dominar los conceptos esenciales de la asignatura. Para asegurarnos de que ese cambio se produce, y que nuestros alumnos se preparan para las clases estudiando los documentos y vídeos que les enviamos y realizan las tareas preparatorias que les proponemos, utilizamos Aplicaciones de cuestionarios online (Google forms para el JITT) y de respuesta personal en clase en tiempo real (Socrative para el TBL y el PI) [7].

El adelanto de la evaluación continua estimula a nuestros alumnos a implicarse en el estudio preparatorio, nos proporciona información sobre sus dificultades y crea el entorno propicio para la introducción de más actividades de aprendizaje activo en las clases [8]. Sin embargo, para tener éxito y mejorar el aprendizaje de nuestros alumnos, esta metodología no sólo requiere de la iniciativa por parte del profesor, sino también de la implicación de los alumnos, que debe ser fomentada y evaluada, para así poder medir los cambios en su trabajo preparatorio, aprendizaje y rendimiento académico que proporcionarán evidencias de la eficacia del cambio metodológico.

En este trabajo pretendemos ofrecer guías a seguir para documentar y evaluar de una manera objetiva y cuantitativa el impacto del cambio metodológico, y para ello, mostramos y recomendamos una serie de procedimientos para realizar estas evaluaciones que pueden ser aplicados en el contexto de la transición entre una enseñanza expositiva tradicional a una enseñanza mediante aula invertida. Los datos usados para ilustrar las metodologías de estimación del impacto metodológico proceden de nuestra experiencia implementando aula inversa adaptativa en cuatro asignaturas de los grados 
de Biología Sanitaria y de Farmacia impartidas en la Universidad de Alcalá. La Tabla I muestra las actividades que realizan el profesor y los alumnos en la metodología de aula inversa adaptativa que hemos aplicado en la impartición de estas asignaturas por las que han pasado entre cinco (Farmacia) y hasta nueve promociones (Biología Sanitaria) de alumnos que han seguido el modelo de aula inversa adaptativa.

Tabla I Metodología del aula inversa en su variante de aula invertida adaptativa

\begin{tabular}{|c|c|c|}
\hline Fase & Actividad de los profesores & Actividad de los alumnos \\
\hline $\begin{array}{l}\text { Antes del } \\
\text { inicio de } \\
\text { cada tema }\end{array}$ & 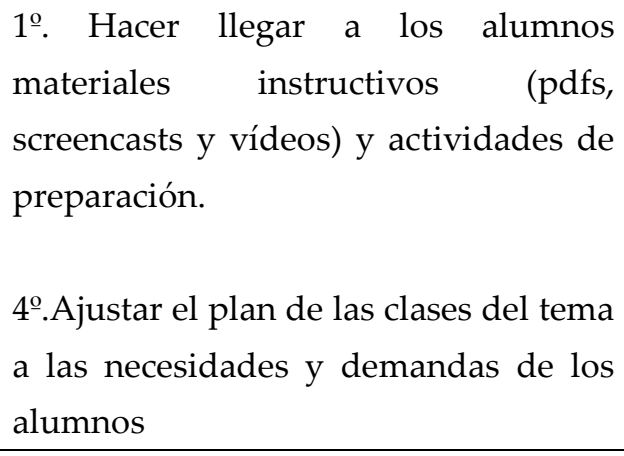 & $\begin{array}{l}2^{\circ} \text {. Realizar el estudio preparatorio de } \\
\text { cada tema y la preparación de cada } \\
\text { sesión ejercicios y notas } \\
\text { 3. }{ }^{\circ} \text { Responder al cuestionario de } \\
\text { reacción a los materiales instructivos, } \\
\text { transmisión de dudas urgentes y de } \\
\text { reflexión sobre lo aprendido }\end{array}$ \\
\hline $\begin{array}{l}\text { Durante las } \\
\text { clases }\end{array}$ & $\begin{array}{l}1^{\underline{0}} \text { Enfocar la clase en la resolución de } \\
\text { dudas y dificultades detectadas: } \\
\text { Peer instruction, intercalar breves } \\
\text { explicaciones con preguntas a los } \\
\text { alumnos y actividades de aprendizaje } \\
\text { activo. } \\
\text { Discusión de viñetas experimentales y } \\
\text { casos prácticos. } \\
\text { Realización de TBL. }\end{array}$ & $\begin{array}{l}\text { 2.Participación en clase : } \\
\text { Responder a preguntas del profesor, } \\
\text { Plantear sus propias preguntas, } \\
\text { Responder a mini-exámenes de } \\
\text { evaluación formativa (quizzs), } \\
\text { Actividades en pequeños grupos }\end{array}$ \\
\hline $\begin{array}{l}\text { Después de } \\
\text { las clase }\end{array}$ & 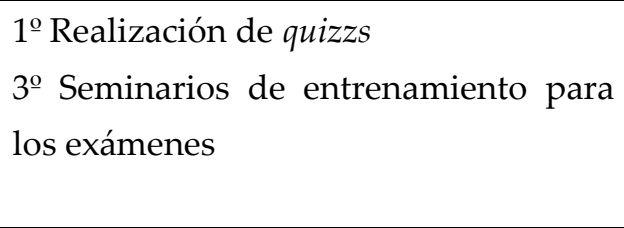 & $\begin{array}{l}2^{\circ} \text { Participación en discusiones sobre } \\
\text { los quizzs } \\
4^{\circ} \text { Reflexión sobre los aprendizajes } \\
\text { adquiridos }\end{array}$ \\
\hline
\end{tabular}

\section{Material y Métodos}

\subsection{Diseño de los estudios de evaluación del impacto del cambio metodológico}

Para evaluar el efecto del cambio, deberemos realizar un estudio de diseño cuasiexperimental en el que comparemos los resultados obtenidos en un grupo en el que hacemos la intervención (aula invertida) y otro control que sigue la contrapartida tradicional (clase expositiva) [9]. Esta opción no era posible en nuestro caso pues debíamos seguir las guías académicas de las asignaturas y debíamos tratar del mismo modo a los alumnos de cada promoción. Por ello optamos por la modalidad de un diseño de control histórico, en el que iniciamos nuestras medidas en una cohorte previa enseñada al modo tradicional, y después las comparamos con las que obtuvimos en las siguientes promociones instruidas mediante aula invertida. Con este modelo de diseño comparativo a partir de una cohorte control histórica en un contexto en el que impartíamos nuestras asignaturas mediante metodología expositiva, deseábamos poner a prueba y comparar otras metodologías alternativas como el aula inversa. Este modelo histórico es la base de muchos estudios de comparación entre aprendizaje activo y enseñanza expositiva tradicional [10] y también de algunos estudios sobre la incorporación del aula invertida [11]. La necesidad de realizar estudios de seguimiento a lo largo de varias promociones de la misma asignatura ya ha sido señalada por otros autores $[12,13]$. 
Dado que la diferencia en rendimiento académico de los alumnos puede depender del profesor que les enseña [14], para evitar esta posible fuente de sesgo en nuestro estudio en cada una de las asignaturas comparadas, las versiones en aula invertida adaptativa de cada asignatura fueron impartidas por los mismos profesores que habían impartido anteriormente la versión tradicional de la misma.

\subsection{Método del aula invertida adaptativa}

Para poner en práctica este método de enseñanza aprendizaje los profesores, antes del inicio de cada tema, enviamos a todos nuestros alumnos materiales instructivos en forma de documentos y videos que nuestros alumnos deben estudiar siguiendo nuestras indicaciones y un cuestionario de reacción a los materiales instructivos en los que los alumnos nos informan de sus dificultades de comprensión, dudas acuciantes, aspectos que les resultan más interesantes así como del tiempo invertido en el estudio y realización de tareas de preparación. Con esta información el profesor adapta su plan de clase a la resolución de dudas manifestadas y a la superación de las dificultades detectadas gracias al trabajo preparatorio y a las respuestas de sus alumnos a los cuestionarios de reacción a los materiales $[1,3]$.

\subsection{Medidas realizadas}

Para evaluar el impacto de la incorporación del aula invertida se realizaron medidas sobre la implicación de los alumnos en el estudio preparatorio (porcentaje de alumnos que realizan más del 505 de las tareas y porcentaje medio de tareas realizadas por cada alumno), Resistencia de los alumnos al cambio metodológico (porcentaje de alumnos que no realizan al menos el $50 \%$ de las tareas encomendadas), tiempo de trabajo de los alumnos, tasas de éxito y de fracaso en la evaluación ordinaria de las asignaturas, calificaciones medias en las pruebas de evaluación del aprendizaje. Finalmente, también se realizaron encuestas para conocer la opinión de los alumnos sobre el cambio y se utilizaron datos de las encuestas oficiales de valoración de la opinión de los alumnos sobre la docencia.

2.4. Estimación del tamaño de efecto (TE) sobre la calificación media en las pruebas de aprendizaje de la asignatura

El TE suele utilizarse para medir el efecto de una innovación metodológica sobre la calificación media de los alumnos en las pruebas de evaluación del aprendizaje en una asignatura. El TE se calcula dividiendo el cambio observado (incremento en el valor medio) en la variable por el valor de la desviación estándar inicial obtenida para esa variable con la metodología tradicional (cohorte de control histórico) y se expresa como g. El valor de un TE de un g equivale a un cambio de magnitud igual al de la desviación estándar de esa variable medida en la población control (histórico en nuestro caso) estudiada en ausencia de la intervención.

\subsection{Grupos estudiados, representación de datos y Análisis estadístico}

Para ilustrar ejemplos de los distintos métodos de medida utilizamos datos de cuatro asignaturas en las que hemos puesto en práctica el aula inversa adaptativa. En cada asignatura hemos estudiado como cohorte control a la última promoción a las que se enseñó con metodología expositiva tradicional y como cohortes experimentales una serie de promociones sucesivas en las que se implementó el modelo de aula inversa.

En algunos casos se representan datos de una determinada promoción de una asignatura. En otras se muestran datos de seguimiento de varias promociones en estudios longitudinales de una asignatura. Finalmente, para integrar resultados de varias asignaturas se emplea el valor de la mediana obtenida al agregar los datos procedentes de las cuatro asignaturas. Los estudios de correlación se han hecho utilizando el Spearman rank correlation test con el programa sigma plot. 


\section{Resultados}

\subsection{Implicación de los alumnos en la realización de las tareas preparatorias}

Al implementar metodología de aula inversa, los alumnos deben responsabilizarse de la primera interacción con la materia a aprender, y, por tanto, el primer desafío de esta metodología es facilitar y guiar el cambio de hábitos de estudio, para así lograr que los alumnos trabajen de un modo distinto a la recepción pasiva de las exposiciones orales por el profesor a las que están acostumbrados en la docencia transmisiva tradicional [15]. A lograr este objetivo contribuye proporcionar incentivos en la calificación de la asignatura para los alumnos que realicen el trabajo preparatorio y los comuniquen respondiendo a cuestionarios de comprobación [16-18]. Estos cuestionarios online son una herramienta esencial que estimula y dinamiza el trabajo preparatorio de los alumnos y permite su observación y control por parte del profesor $[3,7,8]$.

Las respuestas a los cuestionarios nos aportarán información sobre cuáles son los alumnos que realizan las tareas, y esta información la podemos usar además de para bonificar el trabajo de nuestros alumnos, para monitorizar el grado de implicación estudiantil que hemos logrado. Estos cuestionarios diagnósticos de comprobación del estudio preparatorio son una oportunidad para que los alumnos reflexionen sobre lo que han aprendido en los documentos y vídeos instructivos [19]. Las respuestas de nuestros alumnos también nos aportan información muy valiosa para conocer las dificultades, conceptos erróneos y necesidades de aclaraciones, y también nos pueden aportar información sobre cómo se podrían mejorar los materiales instructivos, e incluso (si el cuestionario contiene las preguntas apropiadas), sobre el tiempo de trabajo no presencial que nuestros alumnos dedican a la preparación previa.

El grado de implicación de los alumnos en las tareas preparatorias es el primer aspecto importante a medir para monitorizar la implicación y el grado de seguimiento por parte de los alumnos de la metodología que les proponemos. Por implicación entendemos el tiempo y la energía que los alumnos dedican a la realización de las tareas indicadas por los profesores [20-21], y para el éxito de este modelo, es clave la realización de las tareas preparatorias antes del inicio de los temas y las clases [22]. Procesando la información que nos aportan los formularios de comprobación del estudio previo sobre el número de alumnos que preparan cada tema, y también sobre los alumnos que no lo hacen, podemos cuantificar la proporción de alumnos que se implican en la preparación de las clases y durante cuánto tiempo lo hacen [8].

La implicación de los alumnos de una clase puede estimarse con dos parámetros que miden la proporción de los alumnos que realizan las tareas encomendadas. El primero es el porcentaje de alumnos que realizaron satisfactoriamente una tarea o un conjunto de tareas. El segundo es el porcentaje de alumnos que cumplimentaron satisfactoriamente al menos la mitad de las tareas encomendadas en la asignatura (que equivaldría a obtener al menos una calificación de cinco o aprobar en la evaluación continua correspondiente a esa parte de la asignatura).

Estos dos parámetros, a pesar de que no miden exactamente lo mismo, suelen dar resultados muy similares, aunque sus valores difieren más en los casos extremos cuando o bien, la inmensa mayoría o un número muy reducido de los estudiantes realizan las tareas encomendadas. En el caso extremo de que casi todos los alumnos realicen la mayoría de las tareas el porcentaje de alumnos que realizan al menos el 50\% de las tareas encomendadas puede llegar a ser del $100 \%$, mientras que, el porcentaje medio de tareas que han realizado difícilmente superará el $90 \%$. Esta diferencia se debe a que todos los alumnos (el 100\% de ellos) pueden realizar más de la mitad de las tareas, sin embargo, el porcentaje medio de tareas que realiza cada alumno será casi siempre inferior al $100 \%$.

En la Figura 1 se muestra el resultado medio de estas medidas a lo largo de sucesivas promociones de alumnos en cuatro asignaturas en las que se implantó el modelo de aula invertida.

Cuando el aula inversa se implementa por primera vez (primera promoción) en una asignatura, tanto el porcentaje de alumnos que realiza satisfactoriamente más de la mitad de las tareas preparatorias como el del porcentaje medio de tareas realizadas son bajos $(<50 \%)$, pero en la segunda promoción, suelen ya superar el $50 \%$, en la tercera lo superan ampliamente, en la cuarta promoción suele estar entre el 70 y $90 \%$, y en años sucesivos oscilan entre el 80 y el 100\%. Obsérvese que cuando 
los porcentajes de cumplimentación son muy altos, el porcentaje de alumnos que realizaron más del $50 \%$ de las tareas supera ligeramente al porcentaje medio de tareas realizadas. Aunque no están desglosados en la Figura 1 los porcentajes de alumnos que realizan más del 50\% de las tareas en asignaturas de distintos cursos, estos suelen ser sensiblemente superiores en asignaturas de últimos cursos del grado (90-95\%) respecto a las de primer curso (80-90\%). Nótese que es en la $3^{\underline{a}}$ promoción cuando se alcanzan porcentajes de cumplimentación próximos al 80\%.

Para contabilizar la asistencia a clase llevamos hojas de firmas en las que los alumnos asistentes firman. Para medir la participación de los alumnos en clase llevamos a clase hojas en las que el propio docente o los mismos alumnos se apuntan un positivo tras sus intervenciones. Al realizar estas actividades de control al inicio del cuatrimestre transmitimos a nuestros alumnos el mensaje de que queremos que asistan a las clases y participen en ellas. Esta sección puede dividirse en sub-apartados. Debe proporcionar una descripción concisa y precisa de los resultados, su interpretación, así como las conclusiones experimentales que se pueden extraer.

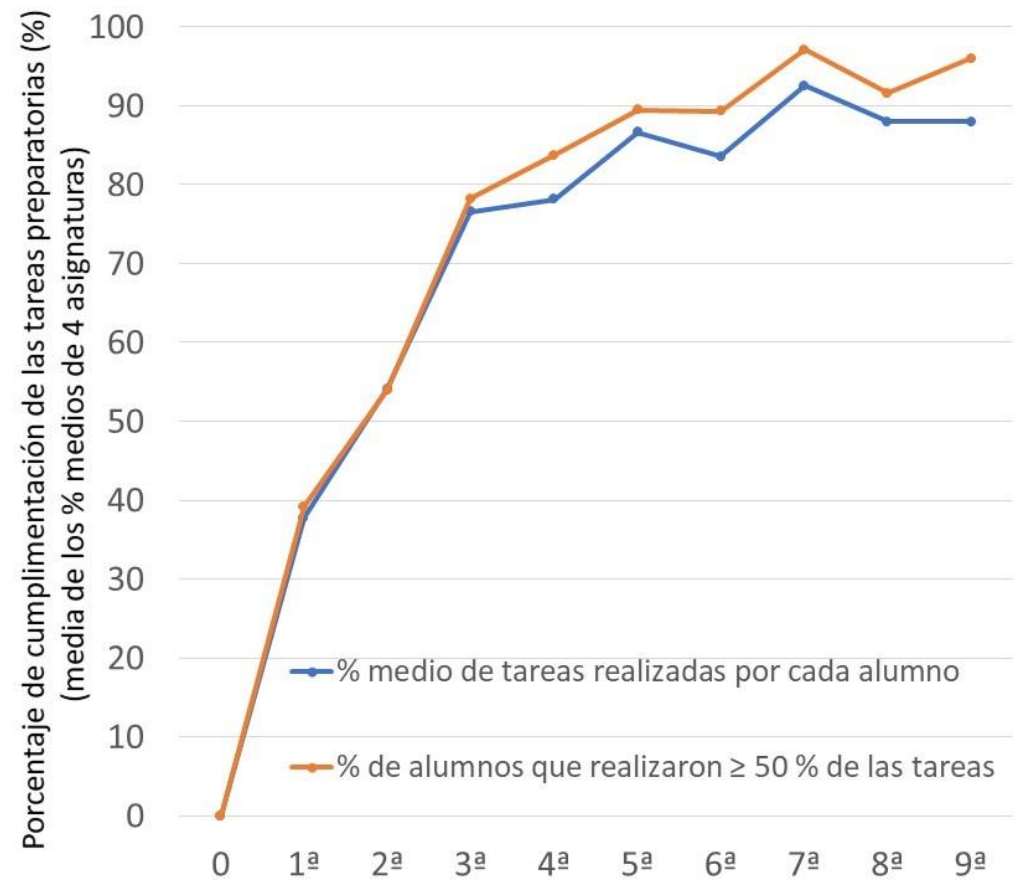

Orden de la promoción en la que se ha utilizado aula invertida

Figura 1 Estimación del grado de implicación de los alumnos en la realización de las tareas preparatorias del aula inversa. Los datos representan la mediana obtenida a partir de los valores medios de cuatro asignaturas impartidas en los grados de Biología Sanitaria y de Farmacia por un grupo de profesores del Área de Inmunología de la Universidad de Alcalá en las que se ha implementado el aula invertida.

\subsection{Resistencia de los alumnos al cambio metodológico}

La falta de implicación de los alumnos en las actividades preparatorias previas a las clases es el principal desafío a la hora de implementar el modelo de aula inversa. Por ello, la resistencia de los alumnos ante el cambio metodológico propuesto, es un parámetro de gran importancia no sólo para valorar el impacto del cambio sino para detectar qué porcentaje de alumnos no se han preparado, y en su caso, tomar medidas para reducirlo, por ejemplo, retrasando determinadas actividades hasta que las hayan preparado más alumnos. Esta resistencia puede ser cuantificada de manera muy sencilla midiendo el porcentaje de alumnos que no llegan a realizar el $50 \%$ de las tareas de preparación que se les proponen. Cuando el porcentaje de alumnos que no se han preparado para la clase es alto, estos alumnos no preparados serán reacios a participar en las actividades de clase y por 
ello es esencial a corto plazo adaptar las actividades de cada clase al porcentaje de alumnos que realizan el estudio, y a largo plazo, lograr que ese porcentaje aumente.

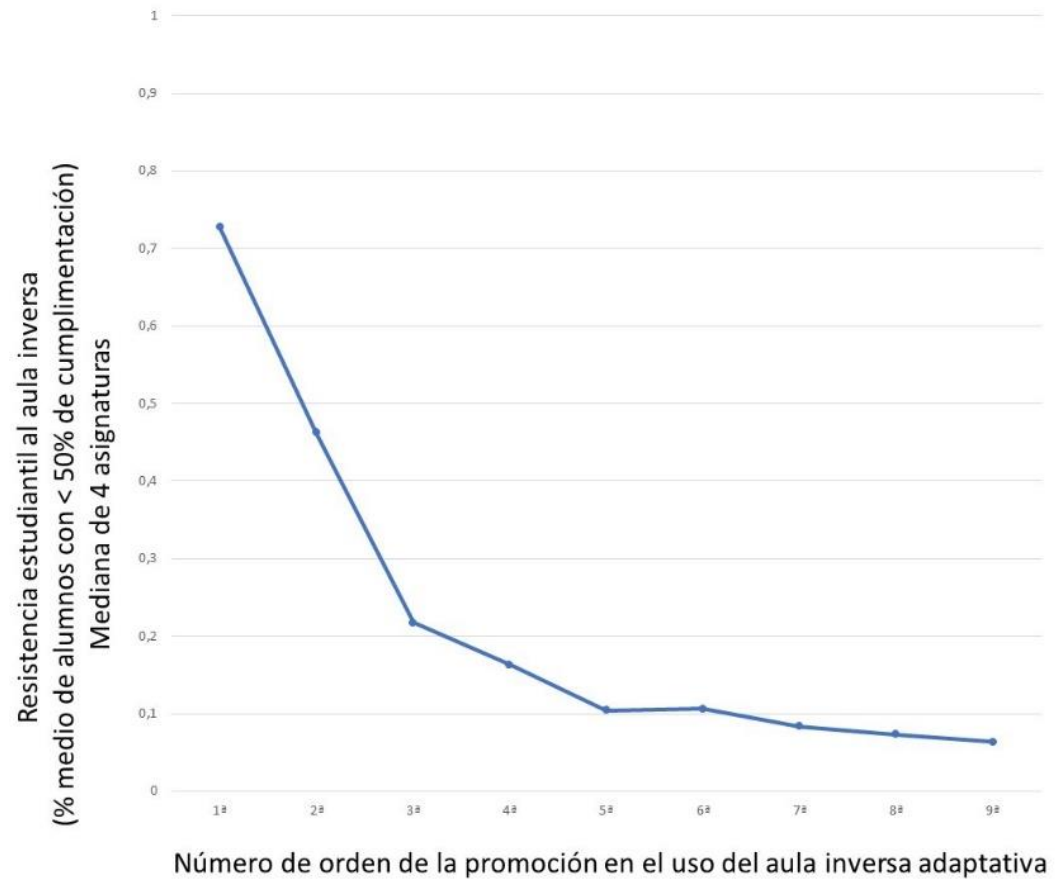

Figura 2 Reducción progresiva de la resistencia del alumnado al cambio metodológico. La resistencia estudiantil se mide mediante el porcentaje de alumnos que no llegaron a realizar la mitad (50\%) de las tareas encomendadas. Se representa la mediana de los valores medios de cuatro asignaturas impartidas en los grados de Biología Sanitaria y de Farmacia en las que se ha implementado el aula invertida.

Típica y lógicamente esta resistencia es máxima en el primer año de implantación del aula inversa en una asignatura, pero se va reduciendo progresivamente hasta hacerse igual o menor al $10 \%$ a partir de la $5^{a}$ promoción. Hemos observado que la resistencia inicial es mayor en asignaturas de los primeros cursos del grado y también en titulaciones con notas de acceso menos exigentes. Reducir cuanto antes el porcentaje de alumnos que no llegan a realizar al menos la mitad de sus tareas es el objetivo de los profesores en los primeros años de implementación. Se trata de acelerar un proceso de cambio cultural desde la primera promoción en la que ponemos en práctica el aula inversa en la que el porcentaje medio de alumnos que pretenderán aprobar sin estudiar antes de las clases inicialmente será mayoritario $(<70 \%)$, hasta que logremos que sea bajo $(<10 \%)$

Gracias a nuestra experiencia adquirida en múltiples asignaturas en las que hemos implementado el aula inversa, hemos aprendido que, la publicidad inicial de la metodología en las clases de presentación de las asignaturas y la recompensa del esfuerzo realizado con bonificaciones o con la asignación de un porcentaje de la calificación de la evaluación continua, son claves para lograr motivar a más alumnos a realizar las tareas preparatorias. Con estas medidas se consigue que un alto porcentaje de alumnos empiece a realizar el trabajo preparatorio. Para lograr que ese esfuerzo se mantenga a lo largo del cuatrimestre es necesario que los alumnos perciban que sus profesores nos tomamos en serio su trabajo preparatorio y que sus intereses y necesidades son considerados a la hora de hacer un rediseño just in time de las actividades en clase $[3,6,7]$. En la metodología just in time teaching, el profesor rediseña su clase tras recibir las reacciones de sus alumnos a la interacción con los materiales instructivos proporcionados, para enfocarla en las dificultades y necesidades que percibe tras analizar las respuestas de sus alumnos. 
En la Tabla II se especifican una serie de acciones recomendadas a fin de lograr motivar a los alumnos para realizar las tareas, y así, superar la resistencia a implicarse más en el aprendizaje de la materia. La implementación de todas estas medidas desde el primer año en el que implantemos el aula inversa consigue reducir el tiempo necesario para lograr que la mayoría de los alumnos se acostumbren a estudiar la mayoría de los temas.

Tabla II Estrategias para vencer la resistencia inicial de los alumnos y aumentar su nivel de implicación.

\begin{tabular}{|l|l|}
\hline Estrategia & Modo de ponerla en práctica \\
\hline $\begin{array}{l}\text { Estimular la } \\
\text { motivación } \\
\text { profunda en el } \\
\text { alumnado }\end{array}$ & $\begin{array}{l}\text { Con una narrativa de desarrollo competencial que muestre cómo } \\
\text { adoptar un papel activo les ayudará a desarrollar su talento y sus } \\
\text { valiosas competencias profesionales }\end{array}$ \\
\hline $\begin{array}{l}\text { Recompensar su } \\
\text { esfuerzo }\end{array}$ & $\begin{array}{l}\text { Establecimiento de bonificaciones a cambio de la realización de las } \\
\text { tareas preparatorias }\end{array}$ \\
\hline $\begin{array}{l}\text { Penalizar al que no } \\
\text { se esfuerce }\end{array}$ & $\begin{array}{l}\text { Asignar en la guía de la asignatura un porcentaje a la realización de las } \\
\text { tareas (los que no lo hagan lo pierden) }\end{array}$ \\
\hline $\begin{array}{l}\text { Publicidad de los } \\
\text { efectos del método } \\
\text { de aula inversa }\end{array}$ & $\begin{array}{l}\text { Mostrar calificaciones de subgrupos de alumnos que se prepararon vs. } \\
\text { los que no lo hicieron. Consejos de los alumnos que sacaron matrícula } \\
\text { en cursos anteriores }\end{array}$ \\
\hline $\begin{array}{l}\text { Demostrar que nos } \\
\text { tomamos muy en } \\
\text { serio su trabajo } \\
\text { preparatorio }\end{array}$ & $\begin{array}{l}\text { Reaccionar frente a sus trabajos y respuestas } \\
\text { Otorgando reconocimiento público a los que han trabajado el Hall offame } \\
\text { (muestra los nombres de los alumnos que hicieron las tareas propuestas) } \\
\text { Replanteando la clase en función de las necesidades e intereses que los } \\
\text { alumnos han mostrado (Just in time teaching) } \\
\text { Respondiendo a sus preguntas urgentes } \\
\text { Señalando ejemplos de lo que es ejemplar y de lo que no es aceptable } \\
\text { como evidencia de un estudio preparatorio adecuado }\end{array}$ \\
\hline
\end{tabular}

En cuanto a la evolución intra-cuatrimestral de la tasa de cumplimentación de las tareas durante el transcurso del cuatrimestre, típicamente se parte de valores máximos en los primeros temas del cuatrimestre (a causa de una buena promoción inicial del aula inversa en la presentación de la asignatura) que, progresivamente van disminuyendo y alcanzan un mínimo que suele coincidir con los exámenes parciales de mitad del cuatrimestre de las asignaturas más temidas por los alumnos. 


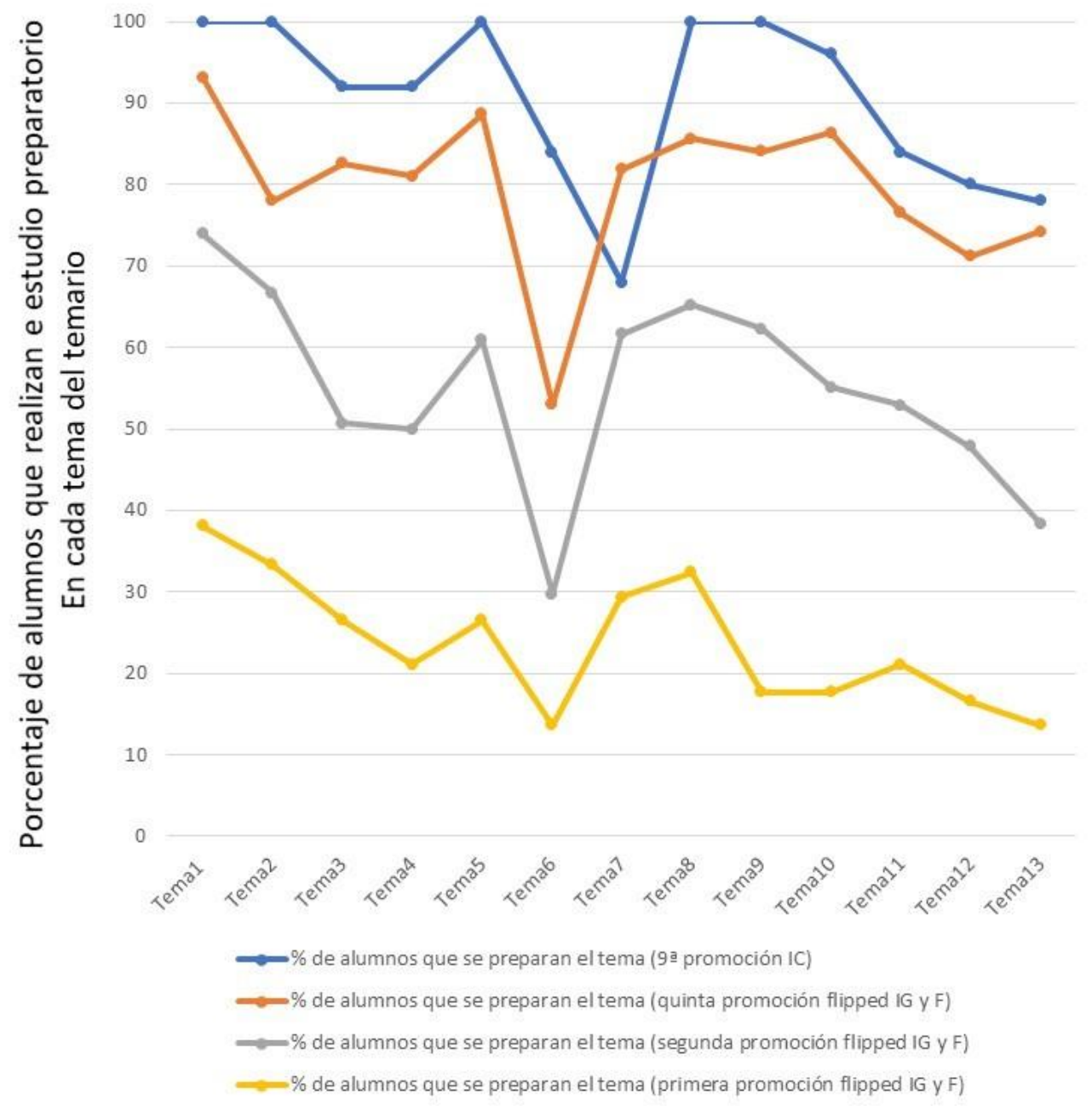

Figura 3 Evolución intra-cuatrimestral del porcentaje de alumnos que realizan el estudio preparatorio de cada tema. Se muestran datos de dos asignaturas, una con un mayor nivel de cumplimentación (Inmunología Clínica, IC) y tres distintas promociones de otra asignatura con menor índice de cumplimentación (Inmunología Genómica y Farmacogenómica, IG y F)

Tras la disminución de cumplimentación delas tareas asociada a estos exámenes parciales, el porcentaje de cumplimentación de las tareas de los siguientes temas se recupera, para volver a caer de nuevo al final del cuatrimestre cuando se inician los exámenes de evaluación continua de final del cuatrimestre. En la Figura 3 puede observarse cómo en la asignatura de Inmunología Genómica y Farmacogenómica (de tercer curso del Grado de Farmacia), los porcentajes han ido aumentando en promociones sucesivas hasta acercarse a los elevados niveles que hemos conseguido en otras asignaturas como Inmunología Clínica (de cuarto curso del grado de Biología Sanitaria).

\subsection{Volumen medio del tiempo de trabajo no presencial del alumno y su relación con el volumen de la carga} de trabajo nominal de los créditos ECTS de una asignatura

Los créditos ECTS establecen el volumen del trabajo que los alumnos deben realizar para aprender lo necesario para superar las asignaturas universitarias [23]. La novedad del crédito ECTS reside, por un lado, en su reconocimiento por las instituciones de educación superior de los países que integran el Espacio Europeo de Educación Superior, y por otro, en la consideración de que el crédito debe incluir un volumen de trabajo presencial, que el alumno realiza en aulas y laboratorios en presencia de los docentes de la asignatura (10 horas por crédito en la mayoría de las universidades españolas), y otro volumen de trabajo no presencial, que el alumno debe realizar estudiando por su cuenta fuera del aula ( 15 horas por crédito en la mayoría de las universidades españolas). 
Los estudios publicados que han comparado la carga ECTS nominal (25 horas/crédito) con el volumen de trabajo real en titulaciones universitarias en España en el ámbito de las ciencias sanitarias y las ingenierías, muestran que, el trabajo medio del alumno por crédito es significativamente inferior a la carga nominal establecida en los créditos ECTS [24-26]. La diferencia es debida a que, aunque las horas de trabajo presencial se cumplen, el número de horas no presenciales de trabajo estudiantil es significativamente inferior al legalmente establecido.

La brecha ECTS, es decir, la considerable diferencia entre la carga de trabajo nominal del crédito ECTS y el menor volumen de trabajo real de los alumnos, debería animar a los profesores universitarios a hacer lo posible para que nuestros alumnos dediquen a su aprendizaje más tiempo fuera de clase. Sólo así los créditos ECTS que ofrecemos en las universidades españolas serán reales, y no una moneda ficticia del aprendizaje universitario sin relación con el tiempo necesario para que se produzca el mismo [3].

Dado que el alumno universitario (según estipulan los créditos ECTS) debe dedicar de media 8 horas de trabajo semanales (sumando el tiempo de trabajo presencial y el no presencial) a cada una de sus cinco asignaturas del cuatrimestre ( $5 \times 8=40 \mathrm{~h} /$ semana), las innovaciones en metodología educativa que implementemos en la educación universitaria deben respetar ese límite de dedicación de tiempo semanal.

Dado que el modelo de aula invertida exige que los alumnos se preparen previamente a las clases, el trabajo preparatorio debe ser diseñado con el fin de que no suponga una carga de trabajo excesiva para los alumnos. Esto puede ser comprobado introduciendo en el cuestionario de comprobación del estudio preparatorio una pregunta del tipo: ¿Cuánto tiempo has empleado en leer los materiales, ver los vídeos, reflexionar y responder a este cuestionario? De esta manera, el profesor obtiene información sobre el tiempo no presencial que los alumnos dedican a prepararse las clases de la asignatura [8].

Gracias al análisis de las respuestas de los alumnos a estas preguntas podemos establecer con precisión y fiabilidad el tiempo que nuestros alumnos emplean en la preparación previa a las clases y cómo distribuyen su tiempo de trabajo entre las distintas tareas encomendadas. Esta información nos permite contrargumentar con hechos a aquellos compañeros que alegan con infundadas conjeturas que el aula inversa producirá una carga docente excesiva para los alumnos.

En las asignaturas en la que empleamos el modelo de aula invertida, el tiempo medio dedicado al trabajo preparatorio para las clases es de $25 \pm 5$ horas en asignaturas de 6 créditos, con una carga total de trabajo no presencial de unas 100 horas. Por tanto, el estudio previo sólo supone para nuestros alumnos un $25 \%$ de la carga de trabajo no presencial correspondiente a esa asignatura de seis créditos ECTS. De esta manera, podemos estar tranquilos porque disponen de otras 75 horas para estudiar después de las clases, y así, prepararse para las pruebas de evaluación y para realizar un trabajo de indagación en pequeños equipos. Del tiempo de trabajo no presencial que nuestros alumnos dedican a la preparación previa a las clases presenciales, algo más de la mitad del mismo lo dedican a leer e intentar comprender los documentos instructivos que los profesores les facilitamos, casi un cuarto de este tiempo, a visionar los vídeos instructivos que les recomendamos, y otro cuarto, a reflexionar y responder a las preguntas reflexivas que les planteamos en los cuestionarios de comprobación del estudio y de detección de dudas y dificultades.

\subsection{Impacto del aula invertida en la tasa de fracaso y en la tasa de éxito en la evaluación ordinaria de una asignatura}

El impacto más importante del aula invertida es el que puede producirse sobre el nivel de aprendizaje adquirido por los alumnos, y en concreto, en relación con su posible aumento de rendimiento académico, el cual podemos definir como el nivel de logro alcanzado por el estudiante en su proceso formativo [27]. Por tanto, una medida objetiva e importante de las repercusiones del cambio metodológico es su repercusión sobre las tasas de éxito y fracaso académico en la asignatura en la que se pone en práctica la innovación. En nuestra experiencia hemos objetivado una disminución de la tasa de fracaso a consecuencia de la puesta en práctica del aula inversa en las distintas asignaturas en las que lo hemos puesto en práctica. 
A modo de ejemplo ilustrativo mostramos los resultados obtenidos en cursos sucesivos en la asignatura de Inmunología Genómica y Farmacogenómica (Figura 4). Gracias a la implementación del modelo de aula inversa en esta asignatura hemos conseguido que la tasa de fracaso en la convocatoria ordinaria descienda desde valores cercanos al $50 \%$ a valores inferiores al $20 \%$. La mejora obtenida ha sido impresionante pues la tasa de fracaso con aula inversa es menor de la mitad de la tasa de fracaso que se obtenía previamente con metodología expositiva tradicional. Sin embargo, esta reducción no se produjo al primer intento, y sólo se empezó a producir a partir del segundo año de implementación de aula inversa, tras lograr superar la fuerte resistencia inicial de una parte mayoritaria de los alumnos ( $>70 \%$ en el primer año) que no realizaron el esfuerzo extra que supone la preparación previa a las clases, ni siquiera en la mitad de los temas.

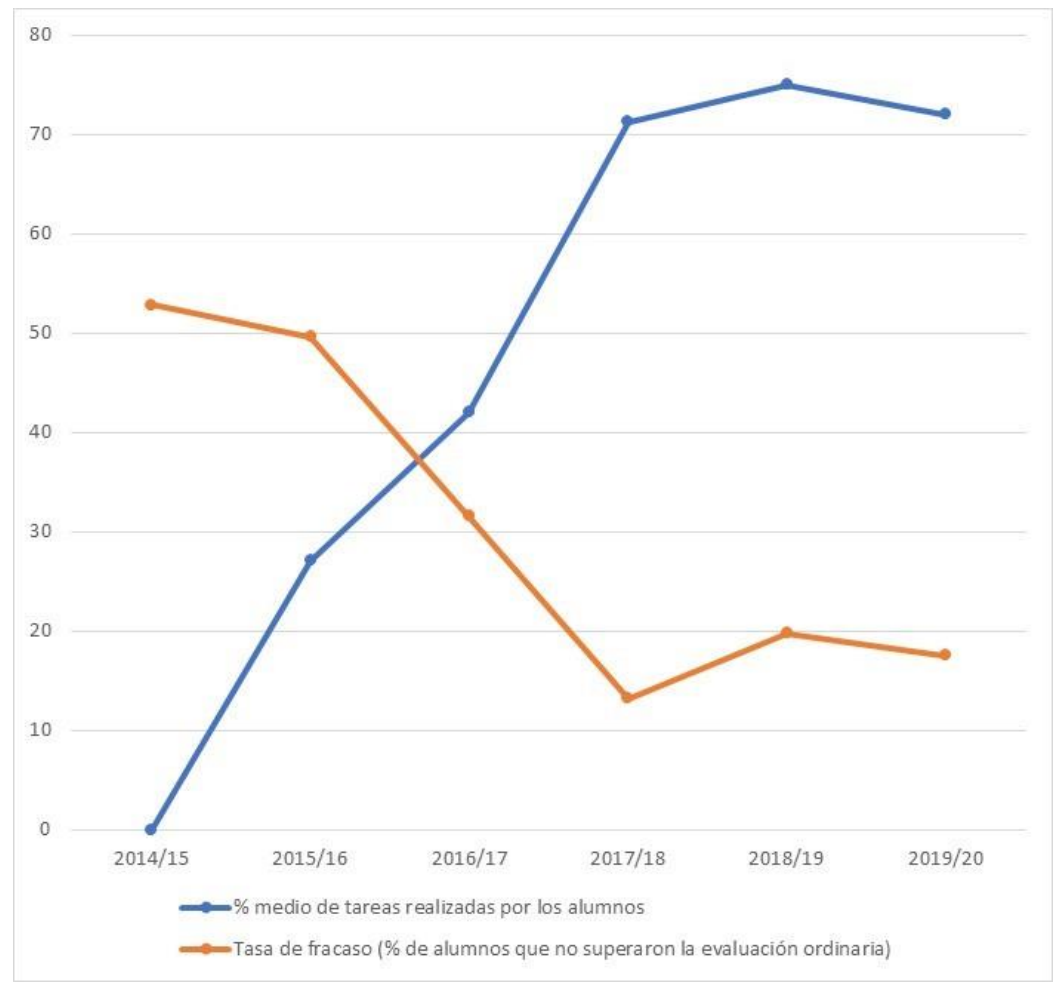

Figura 4 Relación entre la tasa de fracaso de cada promoción de una misma asignatura con el porcentaje de alumnos que realizan estudio preparatorio antes de las clases. La puesta en práctica del aula invertida provoca una reducción de la tasa de fracaso en la evaluación ordinaria de la asignatura Inmunología, Genómica y Fármacogenómica del grado en Farmacia

\subsection{Comparación de los resultados de alumnos con distinto nivel de implicación en el estudio preparatorio:} tasa de éxito en alumnos que preparan distinto número de temas

Con el fin de relacionar el nivel de la implicación de los alumnos en el estudio preparatorio con los resultados académicos y el aprendizaje en el segundo año de implantación del aula invertida en la asignatura de Inmunología, Genómica y Farmacogenómica del grado en Farmacia (curso 2016/17), realizamos un análisis de subgrupos, diferenciando a los alumnos en función del número de temas en los que habían realizado el estudio preparatorio y habían respondido a los cuestionarios de comprobación del estudio. Obtuvimos los resultados mostrados en la figura 5, donde se observa que los subgrupos de alumnos con mayor grado de implicación con su estudio preparatorio, esto es, aquellos que realizaron el estudio preparatorio en un mayor número de temas tuvieron unas tasas de éxito significativamente más altas que los alumnos que prepararon menos temas (Figura 5). Mayores niveles de implicación que se traducen en una realización más frecuente del trabajo preparatorio se relacionan con una mayor probabilidad de aprobar la asignatura en la convocatoria ordinaria. 


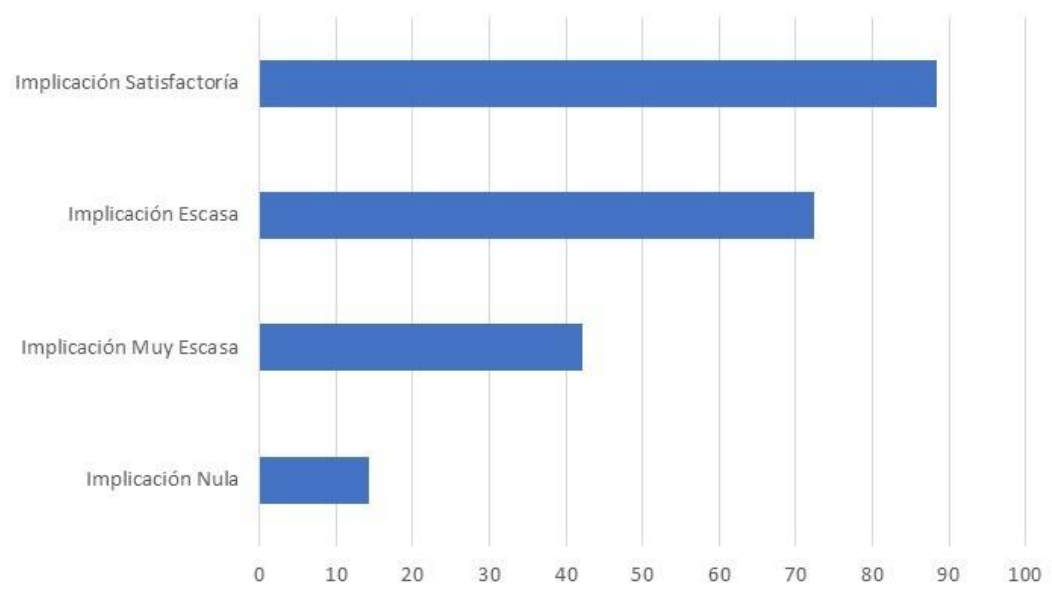

Alumnos que aprobaron la evaluación ordinaria en cada subgrupo (\%)

Figura 5 Relación entre el nivel de implicación en la preparación de la signatura (estimado por el número de temas en los que se realizó el estudio preparatorio y el resultado académico en la asignatura. Los alumnos se dividieron en cuatro subgrupos en función del número de temas que habían estudiado. Implicación Nula: alumnos que no prepararon ningún tema $(n=28)$ Implicación Muy escasa: alumnos que prepararon $<25 \%$ de los temas $(n=38)$ Implicación Insuficiente: alumnos que prepararon entre el 25 y el 50\% de los temas $(n=40)$ Implicación Satisfactoria: alumnos que prepararon más del $50 \%$ de los temas $(n=69)$. La probabilidad de aprobar en la evaluación ordinaria en la cohorte de control histórico enseñada con metodología expositiva tradicional fue del 47,2\% $(n=142)$

Dado que en las asignaturas impartidas mediante aula inversa es conveniente introducir bonificaciones para fomentar el estudio, cabe plantearse la posibilidad de que parte de la mejora observada en la tasa de éxito sea debida al efecto de la bonificación sobre la calificación en la evaluación continua. Para evitar el posible efecto de este factor de confusión, es conveniente evaluar el aumento en la nota de las pruebas de evaluación del aprendizaje antes de sumar la bonificación por el estudio preparatorio (y así lo hacemos en el siguiente apartado). En cualquier caso, siguiendo las recomendaciones publicadas en la literatura [28], el porcentaje máximo de calificación de evaluación continua que asignamos a cambio de la realización de las tareas de preparación es de un $10 \%$ de la calificación de la asignatura. No se recomienda asignar porcentajes de calificación mayores a estas tareas de preparación, pues en caso de hacerlo, se incrementa el riesgo de fomentar la picaresca estudiantil para obtener las bonificaciones mediante atajos en lugar de hacerlo mediante su propio trabajo [28].

Para evitar la posible interferencia del efecto la bonificación en el estudio del efecto del aula inversa, nosotros hemos centrado nuestro estudio en el efecto del aula invertida en los resultados brutos de las pruebas de evaluación del aprendizaje, antes de realizar la adición de las bonificaciones por estudio preparatorio y realización de tareas fuera de clase. Estos estudios del impacto sobre el aprendizaje se muestran en la siguiente sección y se basan en la determinación del tamaño de efecto (TE) sobre la calificación media de los alumnos en las pruebas de evaluación del aprendizaje.

\subsection{Estimación del tamaño de efecto (TE) de una intervención medida relativa del cambio en relación a la desviación estándar de la variable en la cohorte control $(g)$}

El TE es una medida relativa que relaciona la magnitud del impacto de una intervención educativa sobre una variable de resultado en relación a una medida de su variabilidad (la desviación estándar) en la cohorte de control histórico [27]. El TE ha sido utilizado para medir el impacto de intervenciones educativas como el aprendizaje activo $[9,10,29]$ y el aula invertida [5, 27]. El TE permite integrar efectos de un mismo tipo de intervención en distintos contextos y estudios para realizar meta-análisis que reúnan los resultados obtenidos en múltiples estudios realizados en distintas asignaturas y con distintas cohortes de alumnos [27, 30]. En un famoso trabajo [31], Bloom 
estableció que la diferencia en resultados de aprendizaje entre el alumno que aprendía en una clase con respecto al que disponía de un tutor sólo para él era de 2 veces la desviación estándar, es decir de 2 desviaciones estándar (que corresponde a un TE de $2 \mathrm{~g}$ ). En este caso el tutor personal dedicado al aprendizaje de un alumno tendría un TE de $2 \mathrm{~g}$ ). El TE se ha convertido en una manera habitual de evaluar y comparar el impacto de distintas innovaciones educativas entre sí y establecer clasificaciones que permiten conocer que innovaciones tienen mayor impacto sobre el aprendizaje de los alumnos [32].

Muchos estudios originales y varios meta-análisis sobre el impacto del aula inversa en educación superior comparan los resultados obtenidos con metodología tradicional con los obtenidos en el primer año de puesta en práctica del nuevo modelo de aula inversa, y concluyen que, el impacto del aula invertida es positivo y significativo, aunque muy variable y de modesto TE [27,33-37]. Casi todos los estudios publicados en la literatura sobre aula inversa son de corta duración, referidos a la impartición de una asignatura durante un cuatrimestre o semestre [27], y por ello, se echan de menos trabajos que no hagan estudios de corta duración, sino que registren el impacto del cambio metodológico a largo plazo.

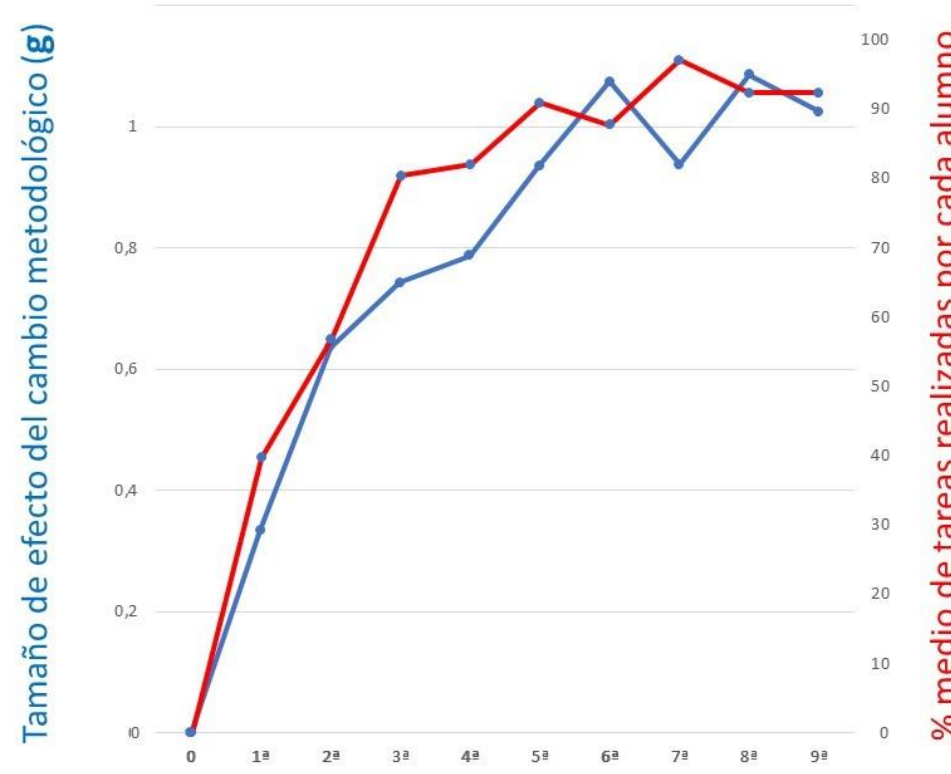

Número de orden de la promoción en la que se aplica el aula inversa

Figura 6 Relación entre el porcentaje de realización de las tareas preparatorias (curva roja) y el tamaño de efecto (TE) observado sobre la calificación media a causa del cambio metodológico al aula invertida (curva azul). Las curvas representan el valor mediana obtenido al agregar los calores medios observados en sucesivas promociones de cuatro asignaturas en las que se implementó el aula invertida. En la curva roja se representan los porcentajes de realización de estudio preparatorio y en la curva azul el tamaño de efecto (TE) sobre las calificaciones en las pruebas de evaluación del aprendizaje

Para saber qué efecto tiene la implementación del aula inversa a largo plazo, deberían hacerse estudios que midiesen el tamaño de efecto en sucesivas promociones para determinar, si el efecto a corto plazo se mantiene en las promociones sucesivas o, si, por el contrario, es transitorio al perderse el efecto novedad de la metodología. Por ello el grupo de profesores del Área de Inmunología de la Universidad de Alcalá, que lleva un total de 9 años utilizando aula invertida, ha realizado un estudio a largo plazo y, gracias a este estudio, hemos comprobado que ciertamente el efecto medio del cambio (en cuatro asignaturas estudiadas) es modesto el primer año, pero sigue aumentando en los cursos siguientes (Figura 6). Posiblemente, esta cinética sea debida a que como ya se vio en la Figura 1, tras el cambio del modelo al aula invertida el porcentaje de alumnos que realizan las tareas preparatorias es mayor cada año, y este aumento progresivo de la implicación, se traduce en una mayor 
participación en las actividades de clase y en un progresivo aumento de su efecto sobre los resultados de aprendizaje. Los resultados en la Figura 6 muestran una alta correlación entre el porcentaje de realización de estudio preparatorio y el TE medido en g de la mejora en aprendizaje.

Cómo se observa en la Figura 6, el efecto del cambio no es inmediato, ni sobre la calificación media, ni tampoco sobre los hábitos de estudio de los alumnos que cursan la asignatura. En el primer año de implementación del aula invertida el TE sobre la calificación media es de 0,3g, en el segundo de $0,6 \mathrm{~g}$, en el tercero y el cuarto se aproxima a $0,8 \mathrm{~g}$ y en promociones sucesivas el TE se mantiene oscilando alrededor del valor de $1 \mathrm{~g}$. El tamaño de efecto correlaciona significativamente con el porcentaje medio de tareas realizadas por los alumnos (Spearman rank order correlation test $\mathrm{r}=0,869$, $\mathrm{p}<0,0000002)$.

Para comprender adecuadamente el significado de los cambios en TE medidos en g es conveniente relacionar lo que supone la mejora de aprendizaje de un g en términos del cambio en percentiles de la distribución de notas de los alumnos. Una subida de valor igual a $1 \mathrm{~g}$ como la que se acaba logrando a largo plazo con el aula invertida, significa un aumento en la distribución de las calificaciones en las pruebas de evaluación del aprendizaje que equivale a una subida del $34,1 \%$ en percentil de las calificaciones de los alumnos en las pruebas de evaluación. Esto significa que con el aula invertida un $84,1 \%$ de los alumnos $(50 \%+34,1 \%)$ superan el nivel de conocimiento que marcaba la mediana con la metodología tradicional, y también que, con aula invertida el $50 \%$ de los alumnos superan el nivel de conocimientos que con la metodología tradicional sólo eran capaces de superar el $15,9 \%$ de los alumnos que obtenían los mejores resultados de la clase.

\section{Estudio de la valoración de la docencia por los alumnos}

La percepción de los alumnos sobre la docencia es subjetiva, pero, pese a ello, podemos cuantificar sus valoraciones y compararlas en distintas promociones, e incluso, compararlas con las que se obtenían cuando se empleaba metodología expositiva tradicional. Los propios alumnos son los mejores jueces para valorar su grado de satisfacción en asignaturas en las que se pone en práctica el aula invertida y compararlo con el que obtienen en las asignaturas impartidas según el modelo expositivo tradicional. Por ello para obtener retroinformación sobre cómo los alumnos valoran la puesta en práctica de una innovación metodológica, podemos pedir que comparen su experiencia con la de otras asignaturas tradicionales [38-40]. Para ello podemos diseñar un cuestionario online como el que puedes encontrar en este link [41].

Link al cuestionario para la valoración del método de aula inversa adaptativa en la asignatura de inmunología clínica en $4^{\circ}$ de Biología Sanitaria de la UAH

Los resultados obtenidos en una promoción de alumnos de inmunología clínica se resumen y comentan a continuación. Los alumnos estaban de acuerdo (al 100\%) con que el estudio preparatorio les había ayudado a comprender los contenidos y a plantear más preguntas en clase, y un $92,4 \%$ se habían sentido más seguros para participar activamente en clase. Con respecto al proceso de garantía del estudio previo, el $100 \%$ estaba bastante o totalmente de acuerdo con que los cuestionarios de comprobación permiten a los profesores conocer las dificultades de los alumnos y responder a ellas. Con respecto a los mecanismos de gamificación, el 100\% estaba bastante o totalmente de acuerdo con que la bonificación del estudio previo les había estimulado a realizar el estudio previo y con que el registro y bonificación de la participación en clase les estimulaba a participar más en clase. Esta respuesta demuestra que en este caso la bonificación fue un estímulo para todos nuestros alumnos aportando motivación extrínseca a los alumnos carentes de motivación intrínseca y reforzando con motivación extrínseca a aquellos que también sienten motivación intrínseca [42]. También el 100\% estaba de acuerdo en que sus interacciones con el profesor habían sido más frecuentes y positivas que en una asignatura tradicional. Finalmente, el $92,4 \%$ de los alumnos consideraban que la incorporación de aprendizaje activo les había ayudado a comprender mejor los contenidos de la asignatura que en una asignatura tradicional. Estas respuestas demuestran que nuestros alumnos mostraban un elevado grado de satisfacción con los cambios que supone la implementación del aula inversa y concuerdan con lo reportado en otros estudios que valoran positivamente las percepciones de los alumnos tras la implementación del aula inversa [38-40,42,43]. 


\section{Promoción y fomento de la cumplimentación por los estudiantes de las encuestas oficiales de evaluación docente}

Es un problema muy extendido en las universidades españolas que las encuestas institucionales de opinión de los alumnos sobre la docencia en las asignaturas son respondidas por porcentajes muy bajos de los alumnos como en el caso de la asignatura de Inmunología, Genómica y Farmacogenómica. Esta situación reduce la fiabilidad de los resultados e introduce sesgos, pues, cuando sólo porcentajes inferiores al 10\% de los alumnos responden a la encuesta la valoración de este grupo muy minoritario de alumnos puede no ser coincidente con la de la mayoría y por tanto no ser representativa de la opinión general.

Nuestras conversaciones con los alumnos nos hicieron sospechar que cuando son pocos alumnos los que responden a una encuesta de evaluación de una asignatura los que los hacen son precisamente los que más insatisfechos y hasta despechados están con el resultado que han obtenido en la evaluación continua de la asignatura (y de esta manera practican el ojo por ojo y castigan al profesor con una mala calificación). Esto nos llevó a hipotetizar que si conseguíamos estimular a mayores porcentajes de alumnos para que se implicasen en la realización de la encuesta oficial de la asignatura la valoración de los profesores mejoraría sensiblemente. Para ello fomentamos con una bonificación de 0,05 puntos la realización de la encuesta oficial (como si esta fuera una tarea más de las que los alumnos realizan a lo largo de la asignatura)

Nuestra solución a este problema ha sido motivar a los alumnos para que realicen la encuesta mediante un mecanismo de comprobación de la realización de la misma. Los alumnos que realizan la encuesta envían una copia de la impresión de pantalla de la aplicación de la encuesta, en la que el sistema les confirma que han realizado la encuesta docente del profesor de la asignatura, a través de un cuestionario cómo el que encontrarás en el siguiente link [44].

Link para confirmar la realización de la encuesta de valoración de la docencia

De esta manera ofrecemos a los alumnos que realizan la encuesta, y nos envían la copia de la pantalla de comprobación, una bonificación en su calificación de evaluación continua similar a la que les ofrecemos por el estudio preparatorio de cada uno de los temas del programa.

La simple implementación de este sistema multiplicó por diez el porcentaje de los alumnos que realizan las encuestas, y por tanto, su resultado es mucho más representativo de la opinión real de la mayoría de los alumnos. En los dos primeros cursos en los que se aplicó este sistema de fomento de la realización de la encuesta, el aumento en porcentaje de alumnos que realizaron la encuesta ascendió espectacularmente desde un 5\% (en la cohorte control histórico) a un 48\% (en la siguiente promoción); y se asoció a un aumento paralelo de la valoración obtenida por los profesores desde un $48 \%$ (en la cohorte control histórico) a un $74 \%$.

El resultado de nuestra estrategia de gamificación denominada pequeña recompensa a cambio de una pequeña acción [45] sirvió para fomentar la realización de las encuestas oficiales mediante una pequeña bonificación de la realización de la encuesta fue que aumentaron espectacularmente tanto el porcentaje de alumnos que realizaron la encuesta (desde valores inferiores al $10 \%$ hasta valores del $50-80 \%$ ) como las valoraciones medias obtenidas por los profesores (desde valores de 40 $50 \%$ a valores superiores al $80 \%$ ). Los datos obtenidos en la asignatura de Inmunología, Genómica y Farmacogenómica del grado de farmacia confirmaron nuestra hipótesis de partida demostrando que existe una correlación directa muy elevada entre el porcentaje de alumnos que responden a la encuesta oficial de valoración y la valoración media obtenida por el profesor (Spearman Rank order correlation test $\mathrm{r}=0^{\prime} 957 \mathrm{p}<0,0000002$ ) (Figura 7). 


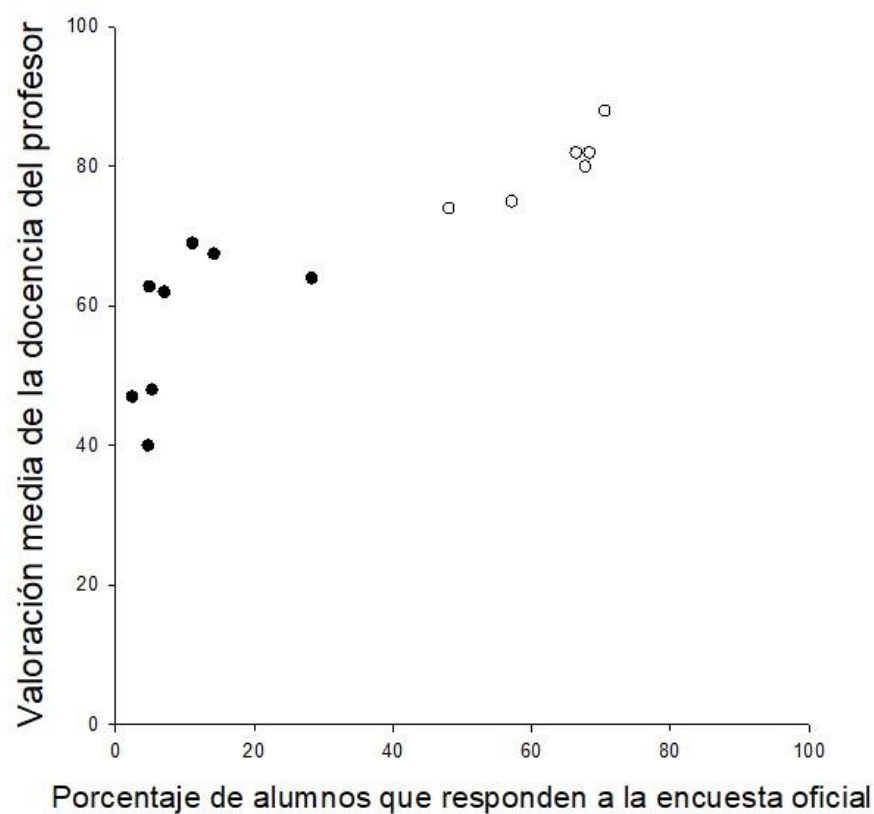

Figura 7 Correlación entre el porcentaje de alumnos que responden a las encuestas oficiales de opinión de los alumnos sobre la docencia y la valoración media de la docencia obtenida en ellas. (•) datos obtenidos en años en los que no se ofreció incentivo por realizar la encuesta (o) datos obtenidos en años en los que sr ofreció un pequeño incentivo por realizar la encuesta oficial de evaluación sobre la docencia

\section{Conclusiones}

Nuestras experiencias de nueve años usando aula invertida en las asignaturas del Área de Inmunología en la Universidad de Alcalá han generado múltiples evidencias de su eficacia. Estas, en su conjunto, demuestran que, si los profesores invertimos un poco más de esfuerzo para implementar el modelo de aprendizaje inverso, y logramos aumentar la motivación de nuestros alumnos por implicarse en el estudio preparatorio y las tareas en el tiempo de clase su aprendizaje, sus calificaciones en los exámenes y su rendimiento académico mejorarán significativamente. Hemos encontrado evidencias de que los subgrupos de alumnos más implicados en el estudio preparatorio previo a las clases (y que por ello realizan con más frecuencia el estudio preparatorio) son los que obtienen mayores tasas de éxito académico en las asignaturas en las que se pone en práctica el modelo de aula inversa adaptativa. Nuestros resultados muestran que, si usamos el aula inversa para hacer prepararse a nuestros alumnos y después enfocar el tiempo de clase en la superación de sus dificultades de aprendizaje, esta metodología fomentará grandes mejoras en el aprendizaje de nuestros alumnos. Esta mejora subraya la importancia de implementar metodologías alternativas a las clases expositivas con el fin de mejorar el rendimiento académico de nuestros alumnos universitarios.

Sin embargo, para lograrlo debemos superar la resistencia de los alumnos a realizar el esfuerzo extra necesario para cambiar sus hábitos de estudio y para implicarse en clases más participativas. Por tanto, es necesario lograr potenciar la motivación intrínseca de nuestros alumnos a emprender esfuerzos que son nuevos para ellos. Debemos usar una narrativa que les haga comprender que el ejercicio de las competencias mediante retos apropiados a su nivel de partida les ayudará a desarrollarlas. También podemos aumentar la motivación extrínseca mediante bonificaciones en la evaluación continua por su trabajo, mostrándoles resultados de los alumnos de promociones anteriores que varían de modo acorde a su implicación con el estudio preparatorio y aportándoles consejos de compañeros que obtuvieron las mejores calificaciones en promociones anteriores [1]. Debemos también ofrecer junto con la exigencia de mayor responsabilidad e implicación en su estudio, un mayor grado de apoyo, enfocándonos en sus dificultades y necesidades, aportándoles 
oportunidades para la evaluación y el feedback formativos. Con todo ello, podemos provocar un cambio en sus hábitos de estudio y actividad mental en clase, que se traducirán en mejora del rendimiento académico y la subsiguiente reducción de la tasa de fracaso, así como una mejora de los resultados en las pruebas de evaluación del aprendizaje. También conseguiremos que mejore la valoración de nuestros alumnos por la docencia recibida y que eso se refleje en las encuestas institucionales de valoración de la docencia.

Este trabajo demuestra que los estudios de seguimiento histórico son esenciales para determinar los efectos a largo plazo de la introducción de nuevos modelos de enseñanza aprendizaje como el aula inversa. Nuestros estudios de seguimiento, de hasta nueve promociones, nos han permitido comprobar que se requieren varios años para cambiar el comportamiento de la mayoría de los alumnos y objetivar el máximo efecto de la nueva metodología cuando ésta es seguida por casi todos los alumnos. La mayoría de los estudios sobre el efecto del cambio desde enseñanza tradicional al aula invertida son de corta duración y sólo miden el cambio tras unas semanas, un cuatrimestre o un curso de duración, asumiendo de hecho, que en ese periodo ya se habrá alcanzado todo el potencial de la nueva metodología. Nuestro estudio demuestra que esta asunción es absolutamente falsa, pues, los efectos g que hallamos en el primer año de implementación del aula invertida son un tercio del valor que los que se alcanzan tras varios años de implementación continuada del modelo de aula invertida.

Las metodologías del estudio de efecto de los cambios en metodología docente propuestas en este artículo nos han permitido cuantificar mejoras significativas en el aprendizaje de nuestros alumnos y niveles elevados de satisfacción con la docencia recibida. Los éxitos que hemos obtenido con el aula inversa nos hacen ser muy optimistas con respecto a sus posibilidades para que su uso se disemine cada día más en educación superior. Si la metodología de aula inversa se extiende ampliamente en las enseñanzas universitarias en España será una oportunidad para cambiar el tradicional modelo de enseñanza transmisivo, basado en la clase expositiva, e introducir mucho más aprendizaje activo y evaluación formativa en nuestras aulas, que ayudarán a que nuestros alumnos adquieran mejores hábitos de estudio y desarrollen competencias. Para ello será necesario que las universidades españolas respalden más y mejor a los líderes en la innovación educativa dentro de sus instituciones y ofrecerles oportunidades para compartir sus experiencias con el aula inversa para que pueda ser adoptada por sus compañeros de centro. Los profesores universitarios deberían considerar la posibilidad de trabajar en equipo, para así, compartir la carga de elaborar los recursos instructivos requeridos para el aula inversa y poder realizar el seguimiento de la preparación de sus alumnos, aprovechándolo para mejorar materiales instructivos [16,46] y actividades de clase [3], dando respuesta de esa manera, a las necesidades de sus alumnos [3].

Agradecimientos: A todos los profesores participantes en el Taller “Cómo poner en práctica el modelo de aula invertida adaptativa", organizado por Joaquín García Estañ y el CEUEM de la Universidad de Murcia, por la inspiración que me aportaron para reunir en un artículo las maneras de evaluar el impacto de la puesta en práctica del aula invertida adaptativa. Este trabajo ha podido realizarse gracias al Proyectos de innovación docente: “Creación de un Repositorio Interuniversitario de Recursos Instructivos y Tecnológicos para la Puesta en Práctica de Asignaturas de Inmunología Básica, Inmunología Clínica e Inmunología Aplicada Basadas en el Modelo De Aula Invertida". Dentro de la "Convocatoria de Proyectos para el fomento de la Innovación en el proceso de Enseñanza-Aprendizaje” de la Universidad de Alcalá, Curso 2017-2018.

Contribución de los autores: Alfredo Prieto Martín concibió el estudio y las intervenciones educativas y realizo el proceso de datos. Alfredo Prieto Martín, David Díaz Martín y Jorge Monserrat Sanz participaron en la enseñanza, la recolección de datos y la evaluación de las asignaturas incluidas en este artículo. Alfredo Prieto Martín, David Díaz Martín y José Barbarroja Escudero escribieron y revisaron el trabajo.

Conflictos de Intereses: Los autores no declaran conflicto de intereses 


\section{Referencias Bibliográficas}

1. Prieto A y Giménez X. La enseñanza universitaria basada en la actividad del estudiante: evidencias de su validez. En Docentes universitarios. Una formación centrada en la práctica Porlan R, de Alba Fernández R. Ed Morata 2020.

2. Prieto A, Díaz D, Monserrat J, Alvarez-Mon M, Sanvicén P, Rinaldi MI. Aula invertida y aprendizaje invertido. En: Las Tecnologías de la Información y la Comunicación como Herramientas Mediadoras de los Procesos Educativos. Volumen I: Fundamentos y Reflexiones. Eds. Ocelli M, García-Romano L, Valeiras N, Quintanilla Gatica M. Santiago de Chile Ed, Bellaterra Ltda. 2018, pp.123-135 Disponible en http://laboratoriogrecia.cl/wp-content/uploads/downloads/2019/01/TIC-LIBRO-Vol-I.pdf

3. Prieto y cols. Aula invertida en enseñanzas sanitarias. Recomendaciones para su puesta en práctica FEM. Revista de la Fundación Educación Médica, ISSN 2014-9832, Vol. 22, №. 6, 2019, págs. 253-262 Disponible en https://www.educacionmedica.net/sec/verRevista.php?id=4447a1368a54257361134

4. Michaelsen LK, Parmelee DX, McMahon KK, Levine RE. Team based learning for health professions education. Stylus Publishing Sterling Virginia 2008

5. Crouch CH, Mazur E. Peer instruction: ten years of experience and results. Am. J. Phys. 2001; 69:970-977.

6. Novak G.,Gavrin A, Christian W, Patterson E. Just-In-Time Teaching: Blending Active Learning with Web Technology. Upper Saddle River. NJ: Prentice-Hall. 1999

7. Prieto A y cols.. (2018). "Nuevas combinaciones de aula invertida con just in time teaching y análisis de respuestas de los alumnos". RIED Revista Iberoamericana Educación a Distancia 21, 175-194 Disponible en http://revistas.uned.es/index.php/ried/article/view/18836

8. Prieto A, Lara I, Díaz D, Monserrat J, Pérez-Gómez A, Vélez J, Otero FM La evaluación del aprendizaje universitario: conflictos entre sus distintas utilidades y compromisos de resolución para preservar las características más esenciales en cada tipo de evaluación RIECS 2018 Vol. 3 Núm. 1 54-64 https://riecs.es/index.php/riecs/issue/view/6

9. Knight, J., Wood, WB. Teaching more by lecturing less. Cell Biol Educ. Winter, 2005; 4(4): 298-310.

10. Freeman, et al. Active learning increases student performance in Science, Engineering, and Mathematics. Proc Natl Acad Sci U S A. 2014; 111(23): 8410-5.

11. Clark KR The effects of flipped model of instruction on student engagement and performance in the secondary mathematics classroom Journal of education online 2015; 12 , 91-115

12. Anderson T, Shattuck J, Design-based research: a decade of progress in education research? Educational Researcher 2012; 4,16-25.

13. Mazur AD, Brown B, Jacobsen M. Learning designs using flipped classroom instruction. Canadian Journal of Learning and technology. 2015; 41, 1-26

14. Gundlach E, Richards KAR, Nelson D, Levesque-Bristol C. A comparison of student attitudes, statistical reasoning, performance, and perceptions for web-augmented traditional, fully online and flipped sections of a statistical literacy class. Journal of Statistics Education 2015; 23, 1-33

15. Betihavas V, Bridgman H, Kornhaber R, Cross M. The evidence for 'flipping out': a systematic review of the fipped classroom in nursing education. Nurse Education Today, 2016; 38 15-21.

16. Prieto A y cols.. "Experiencias de aplicación de estrategias de gamificación a entornos de aprendizaje $\begin{array}{llll}\text { universitario". } & \text { ReVisión, } & \text { 76-92. }\end{array}$ http://www.aenui.net/ojs/index.php?journal=revision\&page=article\&op=viewArticle\&path\%5B\%5D=149

17. Lo CK, Hew KF. A critical review of flipped classroom challenges in K-12 education: possible solutions and recommendations for future research. Research and practice in technology enhanced learning 2017; 12.4

18. Kim MK, Kim SM, Khera O, German J. The experience of three flipped classroom in an urban university: an exploration of design principles. Internet \& Higher Education. 2014; 22,37-50.

19. Schultz D, Duffield S, Rasmussen C, Wageman J. Effects of the Flipped Classroom Model on Student Performance for Advanced Placement High School Chemistry Students J. Chem. Educ. 2014; 91 (9): 13341339.

20. Mosher R McGowan Assessing student engagement in secondary schools: Alternative conceptions, strategies of assessing and instruments. Wisconsin: University of Wisconsin, Research and Development center 1985 .

21. Greene T, Marti CN, Mc Clenney K. The effort-outcome gap: differences for African American and Hispanic community college students in student engagement and academic achievement. The Journal of Higher Education. 2008; 79, 513-19. 
22. Prieto A y cols.. Flipped learning: aplicar el modelo de aprendizaje invertido. Ed. Narcea (Colección Universitaria), Madrid, 2017.

23. ECTS European Credit Transfer System: http://www.eees.es/es/ects [Consultado el 23 de abril 2020]

24. Barjola P, Gómez-Esquer F, González JL, López A, Mercado F, Rivas I. Bordón Crédito ECTS: ¿realidad o ficción? 2011; 63(2): 75-90.

25. Gómez-Esquer F. et al. Cálculo del volumen del trabajo del alumno en las nuevas titulaciones de grado en ciencias de la salud en Evaluación global de los resultados del aprendizaje en las titulaciones dentro del EEES. 2011 Madrid: Dyckinson.

26. Souto-Iglesias A, Baeza-Romero MT. A probabilistic approach to student workload: empirical distributions and ECTS. High Educ. 2018; 76: 1007.

27. Sola T, Aznar I, Romero JM, Rodríguez-García AM. Eficacia del método flipped classroom en la Universidad: Meta-análisis de la producción científica de impacto. REICE Revista Iberoamericana sobre Calidad, Eficacia y Cambio en Educación 2019;17:25-38.

28. James M., The effect of grading incentive on student discourse in Peer Instruction. American Journal of Physics, 2006:74(8): 689-691

29. Hake, R. Interactive-engagement versus traditional methods: A six-thousand-student survey of mechanics test data for introductory physics course. Am. J. Phys. 1998; 66: 64-74.

30. Hedges IV. Distribution theory for glass estimator of effect size and related estimators. Journal of Educational Statistics. 1981; 6(2): 107-28

31. Bloom, B The 2 Sigma Problem: The Search for Methods of Group Instruction as Effective as One-to-One Tutoring. Educational Researcher. 1984; 13 (6): 4-16. doi:10.3102/0013189x013006004.

32. Hattie J. Visible learning: A synthesis of over 800 meta-analyses relating to achievement. 2008 New York: Routledge.

33. Lo CK, Hew KF, Chen G. Toward a set of design principles for mathematics flipped classrooms: A synthesis of research in mathematics education. Educational Research Review. 2017; 22: 50-73

34. Chen KS, Monrouxe L, Lu YH, Jenq CC, Chang YJ, Chang YC, Chai PYC. Academic outcomes of flipped classroom learning: A meta-analysis. Medical Education, 2018; 52(9): 910-924.

35. Crimmins, MT Midkiff B. High structure active learning pedagogy for the teaching of organic chemistry: assessing the impact on academic outcomes Journal of chemical Education 2017; 94: 429-438

36. Cheng L, Ritzhaupt AD, Antonenko P. Effects of the flipped classroom instructional strategy on students' learning outcomes: A meta-analysis. Educational Technology Research \& Development 2018, 1-32.

37. Hu R, Gao H, Ye Y, Ni Z, Jiang N, Jiang X. Effectiveness of flipped classrooms in Chinese baccalaureate nursing education: A metanalysis of ramdom controlled trials International Journal of Nursing Studies. 2018; 79: 94-103.

38. Martínez R, Ruiz MC. Estudiantes motivados y participativos. ¿Una Utopia? No, una realidad gracias al aula invertida. Congreso In-Red Universitat Politécnica de Valencia 2019; 364- 377

39. Murillo-Zamorano LR, López JA, Godoy-Caballero AL. How the flipped classroom affects knowledge, skills and engagement in higher education: Effects on students' satisfaction. Computers \& Education 2019; 141

40. Estriegana R, Barchino R, Medina Merodio J Information and communications technology in a blended course: "The best of two worlds" Proceedings of ICERI2016 Conference 2016 Seville Spain

41. Valoración del método de aula inversa adaptativa en la asignatura de inmunología clínica en $4^{\circ}$ de Biología Sanitaria de la UAH Disponible en https://forms.gle/kkHjFKMGCwtdzZsx8 [Consultado el 23 de abril 2020]

42. Deci E, Ryan R. Intrinsic determination and Self-determination in human behaviour. 1985 New York: Plenum

43. Awidi IT, Paynter M. The impact of a flipped classroom approach on student learning experience. Computers \& Education. 2019; 128: 269-283

44. Link para confirmar la realización de la encuesta de valoración de la docencia. Disponible en https://goo.gl/forms/AdtZbGYDIGm4zuQM2 [Consultado el 23 de abril 2020]

45. Kapp K. The Gamification of Learning and Instruction: Game-based Methods and Strategies for Training and Education. 2012 Pfeiffer.

46. Contreras JA, Arias J, Melo MG, Martin R. Uso del modelo de aprendizaje inverso para mejorar materiales educativos universitarios. Revista Ibérica de Sistemas y Tecnologías de Información 2017; 23:17-32. 
(C) 2020 por los autores; Esta obra está sujeta a la licencia de Reconocimiento 4.0 Internacional de Creative Commons. Para ver una copia de esta licencia, visite http://creativecommons.org/licenses/by-nc-nd/4.0/. 